\title{
Unsteady Pressure Coefficient around an Elliptic Immersion Nozzle
}

\author{
Yoshiaki UEDA, Teruhiko KIDA ${ }^{1)}$ and Manabu IGUCHI ${ }^{21}$
}

Formerly Graduate Student, Department of Energy Systems Engineering, Osaka Prefecture University, 1-1 Gakuencho, Sakai, Osaka 599-8531 Japan, now at Innovation Plaza Hokkaido, Japan Science and Technology Corporation, North 19, West 11, Sapporo 060-0819 Japan. 1) Department of Energy Systems Engineering, Osaka Prefecture University, 1-1, Gakuencho, Sakai, Osaka 599-8531 Japan. $\quad 2$ 2) Division of Materials Science and Engineering, Graduate School of Engineering, Hokkaido University, North 13, West 8, Kita-ku, Sapporo 060-8628 Japan.

(Received on February 16, 2004; accepted in final form on April 26, 2004)

\begin{abstract}
An unsteady molten steel meniscus flow sometimes occurs in the continuous casting mold. When it impinges on the immersion nozzle, a severe vertical pressure difference is induced on the outer surface of the nozzle. Mold powder entrapment near the immersion nozzle is closely associated with the pressure difference. The use of an elliptic immersion nozzle is expected to effectively lower the difference, and accordingly, suppress the mold powder entrapment. The pressure difference around the elliptic immersion nozzle however is not quantitatively known yet. In this study it was numerically calculated by the vortex method over a wide aspect ratio range of the nozzle for two types of unsteady meniscus flows. The validity of the numerical results was confirmed from a comparison with previous results obtained from cold model experiments.
\end{abstract}

KEY WORDS: steelmaking; continuous casting; mold powder entrapment; elliptic cylinder; immersion nozzle.

\section{Introduction}

Suppression of mold powder entrapment into molten steel in the continuous casting mold is of essential importance for producing clean steel. ${ }^{1-3)}$ Many efforts have been devoted to understand the mechanism of the entrapment based mainly on cold model experiments. The following five types of mechanisms have been proposed, ${ }^{3,4)}$ as can be schematically seen in Fig. 1.

(1) Entrapment induced by steady reversing flow.
(2) Entrapment caused by Kelvin-Helmholz instability at an interface between molten steel and molten mold powder.

(3) Entrapment due to Karman vortex streets generated behind the immersion nozzle.

(4) Entrapment caused by attack of large argon bubbles onto the interface between the molten steel and molten mold powder.

(5) Entrapment due to vertical pressure difference along the outer surface of the immersion nozzle.

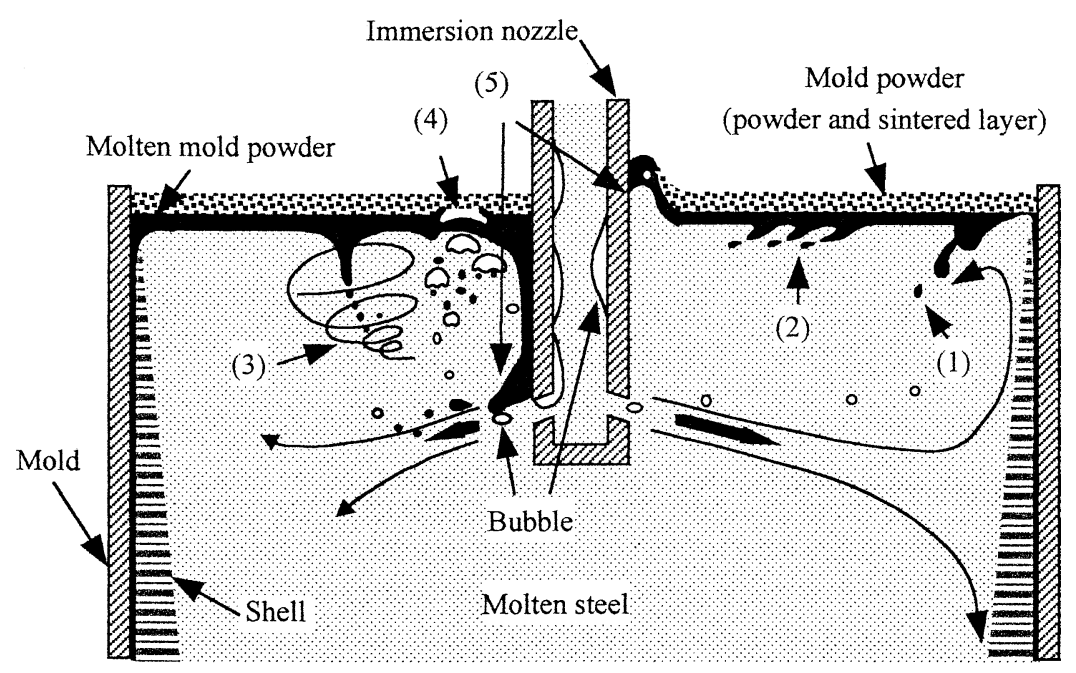

Fig. 1. Schematic illustration of mold powder entrapment. 
One of the present authors ${ }^{4)}$ has pointed out that the fourth and fifth mechanisms are most responsible for the mold powder entrapment in the real continuous casting mold. The use of an immersion nozzle of an elliptic crosssection is beneficial for suppressing the mold powder entrapment due to a vertical pressure difference depicted in the type (5). ${ }^{5}$ ) The applicability of this method was partly confirmed based on water model experiments. Unfortunately, information on the unsteady pressure distribution around an elliptic cylinder and the drag coefficient is not available in literature, ${ }^{6}$ although the drag coefficient, $C_{\mathrm{D}}$, for steady flows is known. ${ }^{7,8)}$ This paper presents numerical data on the unsteady pressure coefficient for an elliptic immersion nozzle of an arbitrary aspect ratio. These data would be useful for designing an optimum immersion nozzle for preventing mold powder entrapment in the real continuous casting mold.

\section{Definition of Pressure Coefficient}

The pressure coefficient for a cylinder, $C_{\mathrm{p}}$, is defined as follows:

$$
\begin{gathered}
C_{\mathrm{p}}=\Delta p /\left(\rho V_{0}^{2} / 2\right) \\
\Delta p=p-p_{\mathrm{s}} \cdots
\end{gathered}
$$

where $\Delta p$ is the pressure difference, $p$ is the pressure on the surface of the cylinder, $p_{\mathrm{s}}$ is the static pressure, $\rho$ is the density of fluid, and $V_{0}$ is the velocity of flow approaching the cylinder.

\section{Description of Flow Field and Calculation Method of Pressure Coefficient}

Figure 2 shows the coordinate system for an elliptic cylinder. The short and long diameters are denoted by $D_{y}$ and $D_{x}$, respectively. In this calculation, $D_{x}$ was increased, while $D_{y}$ was kept constant. The aspect ratio, $A_{\mathrm{s}}$, was defined as $D_{x} / D_{y}$, and set to be 1,2, 3 and 4 . Two types of transient velocities were chosen for approaching flows.

(1) Sudden Acceleration

$$
\begin{array}{ll}
V=0 & \text { for } t<0 \\
V=V_{0} & \text { for } t \geq 0
\end{array}
$$

The approaching flow impinges on the cylinder impulsively. This velocity history states that the velocity of an unsteady reversing flow in the real continuous casting mold changes suddenly due to occurrence of uneven discharge flow from the nozzle ports. Therefore, this is a limiting case appearing in the real mold.

(2) Gradual Acceleration

$$
\begin{aligned}
& V=0 \text { for } t<0 \\
& V=\left(0.12 V_{0} / \pi\right) \tan ^{-1}[(t-3.0) / 1.7+0.35 \pi] \quad \text { for } t \geq 0
\end{aligned}
$$

The history expressed by Eq. (4) was chosen to fit the experimentally measured transient velocity, ${ }^{5)}$ as shown in Fig. 3. The dimensionless time $t^{\prime}$ is defined as

$$
t^{\prime}=t V_{0} / D_{x}
$$

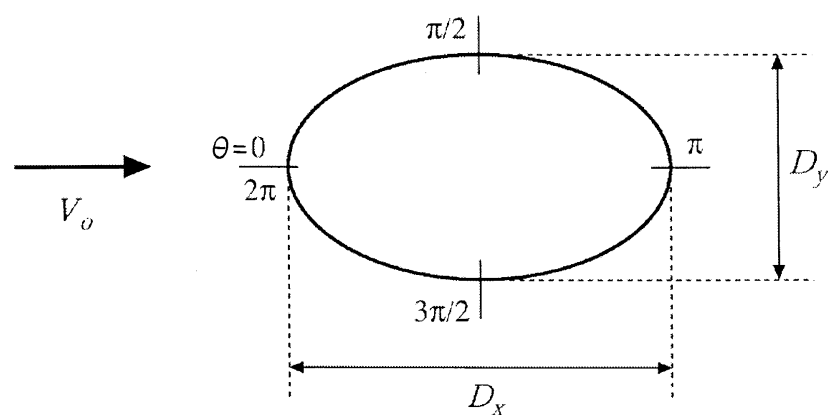

Fig. 2. Coordinate system fixed with the center of the elliptic cylinder.
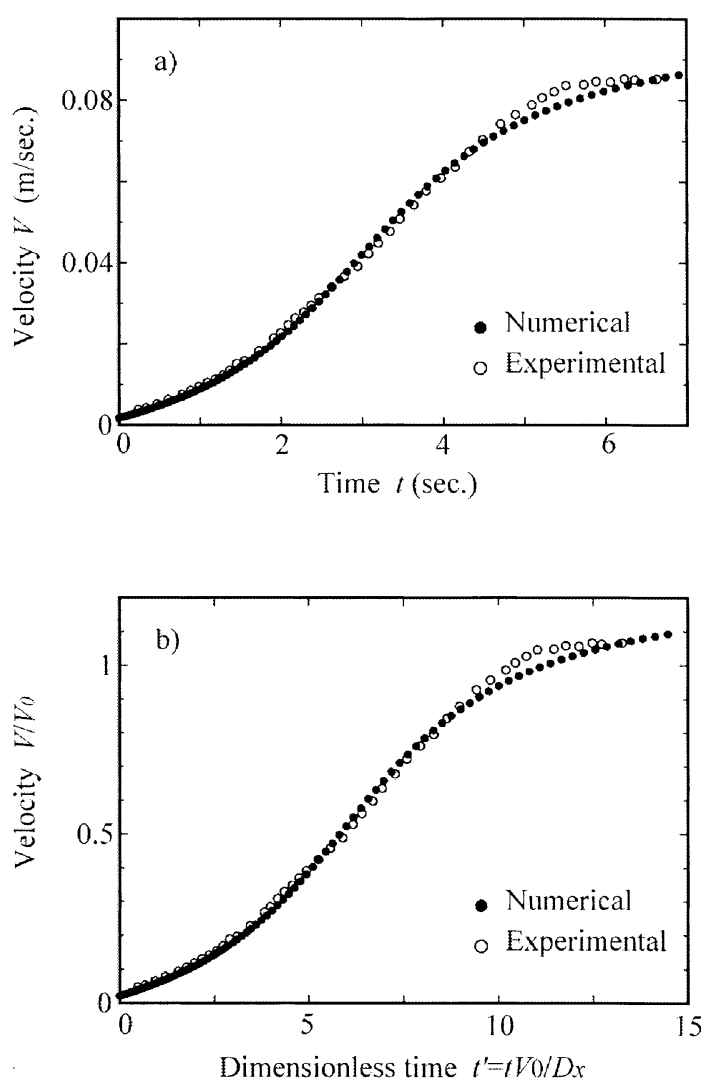

Fig. 3. Transient velocity profile.

where $V_{0}$ is set to be $0.08 \mathrm{~m} / \mathrm{s}$. This velocity history was chosen because the velocity of an unsteady reversing flow in the real mold is considered to be increased gradually.

The governing equations for the flow around an elliptic cylinder were solved by the vortex method.,10) The details of the method are described elsewhere. ${ }^{11,12)}$ The applicability of the vortex method to unsteady flows around a bluff body such as a cylinder was confirmed by Kida et al. ${ }^{11)}$

\section{Calculated Results and Discussion}

\subsection{Pressure Distribution around Elliptic Cylinder}

Figures 4(a) through 4(e) show a calculated result typical of the pressure coefficient, $C_{\mathrm{p}}$, around an elliptic cylinder of $A_{\mathrm{s}}=4$ on the initial stage in the transient period of sudden acceleration case. The pressure coefficient at the forward stagnation point $(\theta=0)$ hardly changed with respect to time. On the other hand, the pressure coefficient at 
ISIJ International, Vol. 44 (2004), No. 8
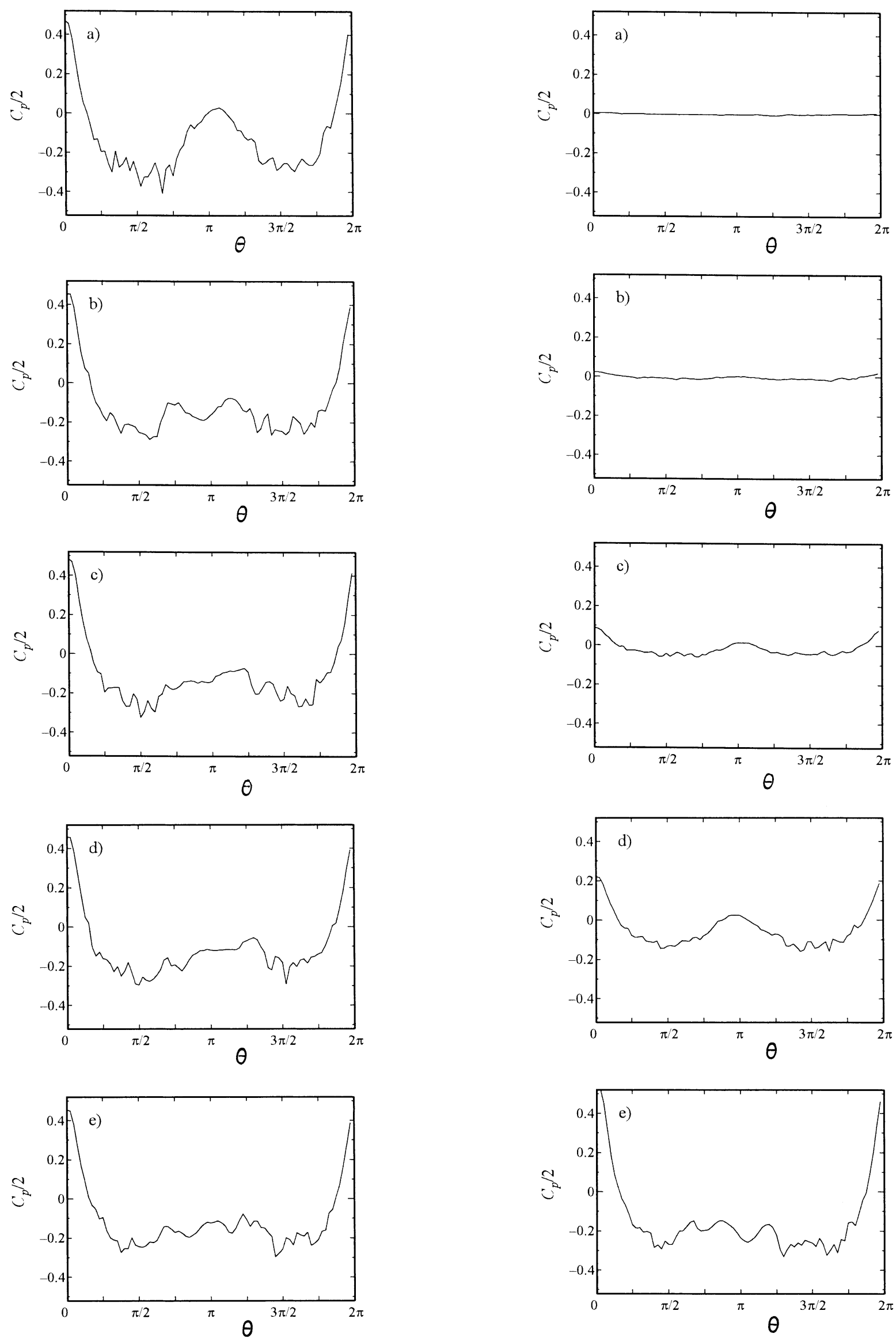

Fig. 4. Pressure coefficient in the case of sudden acceleration and aspect ratio $A_{\mathrm{s}}=4.0$; (a) $t^{\prime}=1.02$, (b) $t^{\prime}=3.0$, (c) $t^{\prime}=5.04$, (d) $t^{\prime}=7.02$, (e) $t^{\prime}=14.04$.

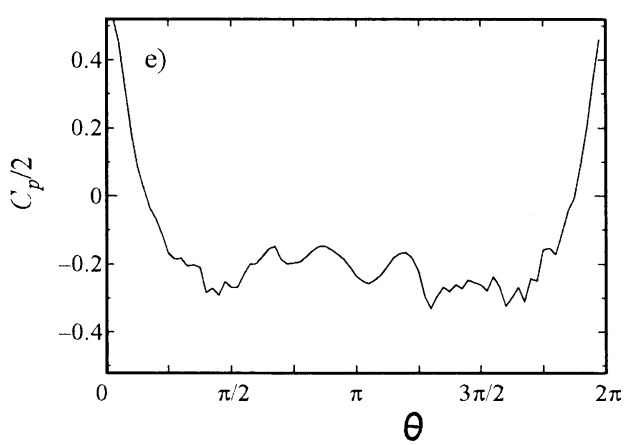

Fig. 5. Pressure coefficient in the case of gradual acceleration and aspect ratio $A_{\mathrm{s}}=4.0$; (a) $t^{\prime}=1.02$, (b) $t^{\prime}=3.0$, (c) $t^{\prime}=5.04$, (d) $t^{\prime}=7.02$, (e) $t^{\prime}=14.04$. 
the rear stagnation point $(\theta=\pi)$ decreased rapidly from 0 to approximately -0.2 and the distribution of $C_{\mathrm{p}} / 2$ on the rear part of the cylinder became nearly flat at $t^{\prime}=14.02$. The minimum value of the pressure coefficient appeared at around $\theta=\pi / 2$ and $\theta=3 \pi / 2$.

Figures 5(a) through 5(e) show the pressure coefficient for the gradual acceleration. The pressure coefficient at the forward stagnation point increased gradually from 0 to approximately 0.6 , while the value at the rear stagnation point decreased gradually from 0 to approximately -0.2 . The minimum pressure coefficient appeared at around $\theta=\pi / 2$ and $\theta=3 \pi / 2$ just as in the case of sudden acceleration. The distribution of $C_{\mathrm{p}} / 2$ on the rear part of the cylinder approached a flat distribution as time elapsed.

\subsection{Maximum and Minimum Pressure Coefficients around Elliptic Cylinder}

Figures 6 and 7 show the value of $C_{\text {pmax }} / 2$ for sudden and gradual accelerations, respectively, where $C_{\mathrm{pmax}}$ is the maximum value of the pressure coefficient appearing at the forward stagnation point. The calculated value of $C_{\mathrm{pmax}} / 2$ for the sudden acceleration almost unchanged with time $\left(C_{\text {pmax }} / 2 \approx 0.5\right)$ but decreased slightly with an increase in the aspect ratio, $A_{\mathrm{s}}$. On the other hand, $C_{\mathrm{pmax}} / 2$ for the gradual acceleration increased with time from 0 to around 0.6 . The value of $C_{\mathrm{pmax}} / 2$ for the gradual acceleration at a dimen-

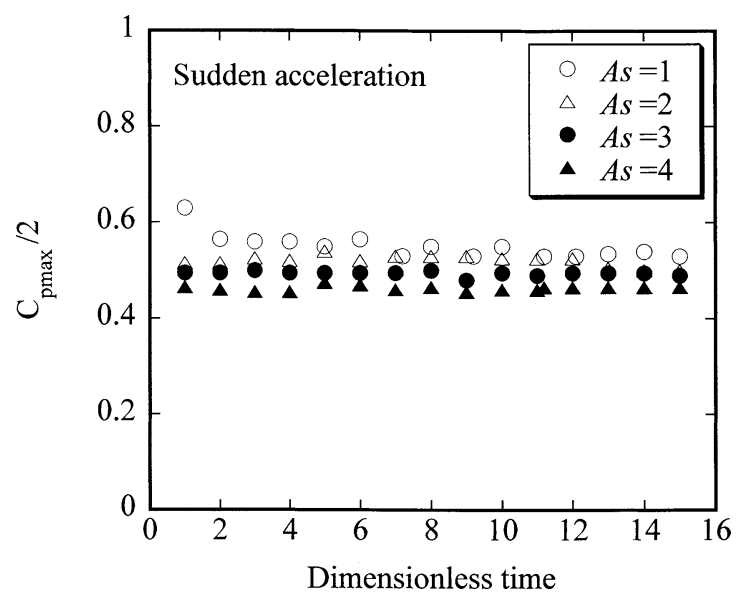

Fig. 6. Relationship between maximum pressure coefficient and dimensionless time for sudden acceleration.

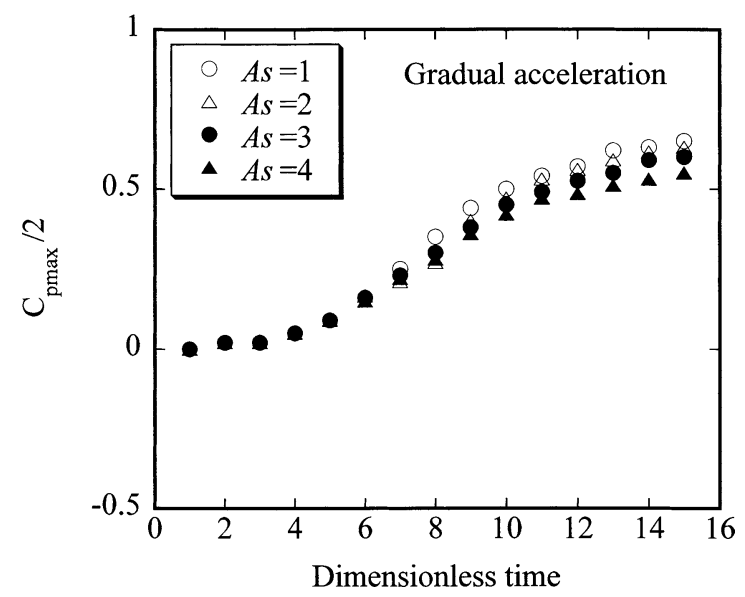

Fig. 7. Relationship between maximum pressure coefficient and dimensionless time for gradual acceleration. sionless time, $t^{\prime}$, of 15 is different from that for the sudden acceleration because the values of $V$ are different with each other.

The calculated values of $C_{\mathrm{pmin}} / 2$ for the sudden and gradual accelerations are plotted in Figs. 8 and 9, respectively, where $C_{\mathrm{pmin}}$ is the minimum pressure coefficient appearing around $\theta=\pi / 2$ and $\theta=3 \pi / 2$. The value of $C_{\mathrm{pmin}} / 2$ for the sudden acceleration changed in a somewhat complex manner with a lapse of time unlike $C_{\mathrm{pmax}} / 2$. When the aspect ratios were 3 and 4 , the value of $C_{\mathrm{pmin}} / 2$ for the sudden acceleration and that for the gradual acceleration approached with each other. On the other hand, a significant difference was seen between them for $A_{\mathrm{s}}=1$ and 2. Figure 10 shows the calculated values of $C_{\text {pmin }} / 2$ for the gradual acceleration which are re-plotted from Figs. 8 and 9. The minimum pressure coefficient decreased monotonically with time, and it approached zero as the aspect ratio increased.

Experimental results for unsteady pressure coefficients on two-dimensional bodies are very limited even for a circular cylinder. According to a previous study of flow around an impulsively started circular cylinder, ${ }^{13)} C_{\mathrm{pmin}} / 2$ is approximately -1.25 at the rear part of the cylinder. This value is approximately the same as that shown in Fig. 8 for a circular cylinder $\left(A_{\mathrm{s}}=1\right)$ subjected to sudden acceleration. This fact partly supports the adequacy of the present numerical calculation.

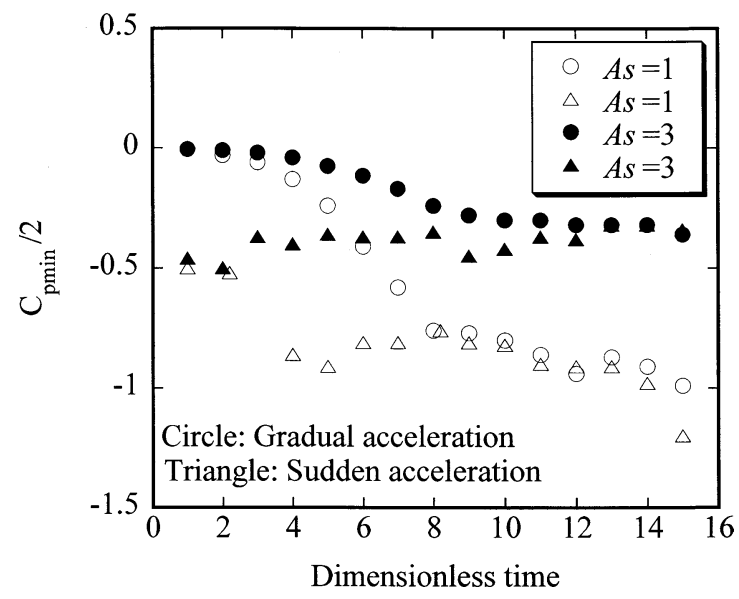

Fig. 8. Relationship between minimum pressure coefficient and dimensionless time for aspect ratios of 1 and 3 .

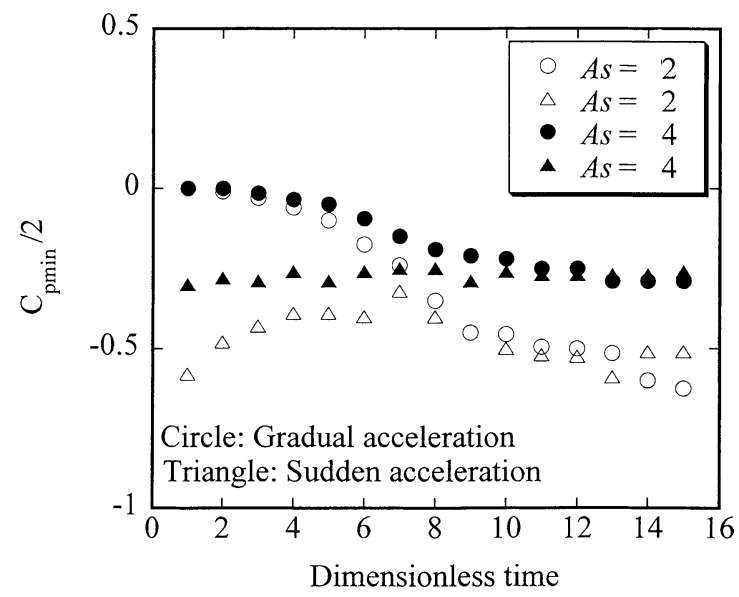

Fig. 9. Relationship between minimum pressure coefficient and dimensionless time for aspect ratios of 2 and 4. 


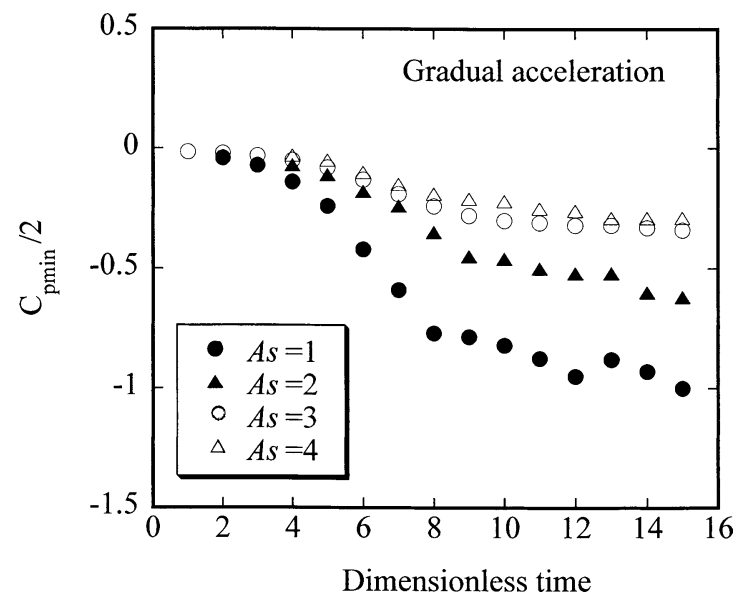

Fig. 10. Relationship between minimum pressure coefficient and dimensionless time for gradual acceleration.

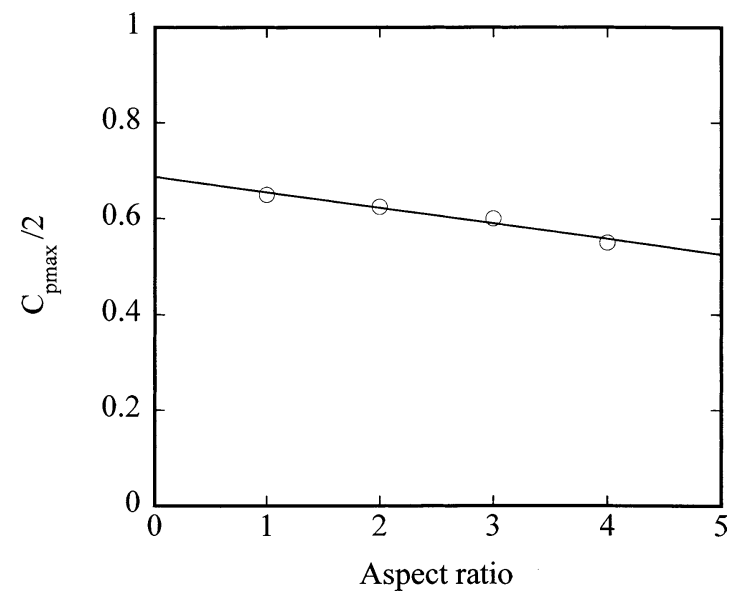

Fig. 11. Relationship between maximum pressure coefficient and aspect ratio for gradual acceleration at $t^{\prime}=15$.

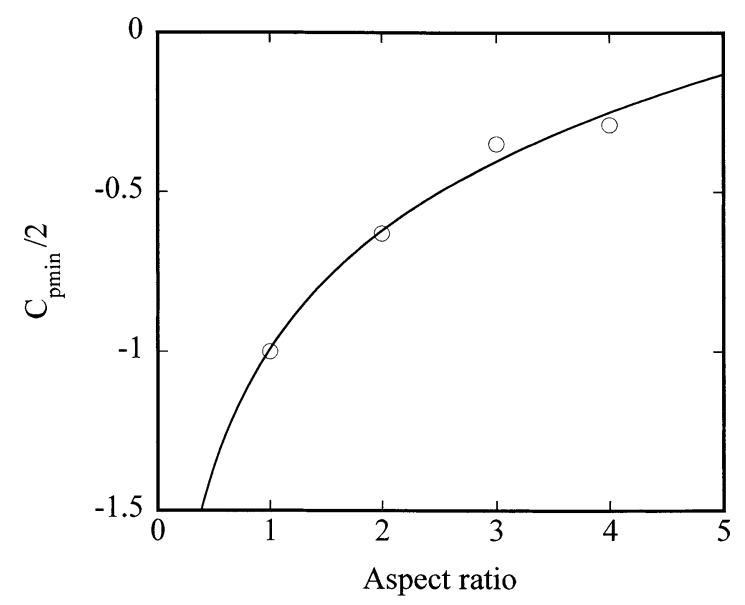

Fig. 12. Relationship between minimum pressure coefficient and aspect ratio for gradual acceleration at $t^{\prime}=15$.

The maximum and minimum values of the pressure coefficient for the gradual acceleration at a dimensionless time of 15 are summarized in Figs. 11 and 12, respectively. The solid line in each figure was drawn so as to pass through the calculated values as close as possible. The values of $C_{\mathrm{pmax}} / 2$ and $C_{\mathrm{pmin}} / 2$ for an arbitrary aspect ratio other than $1,2,3$, and 4 can be evaluated from these two figures. The maximum pressure coefficient decreased linearly with respect to

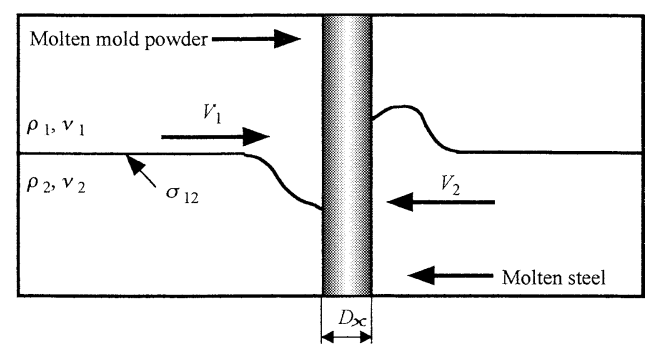

Fig. 13. Flow field and some symbols used in this study.

the aspect ratio. The decreasing rate is small, while the minimum pressure coefficient changed drastically with $A_{\mathrm{s}}$. The data will be used in a later section for calculating the maximum penetration depth of molten mold powder.

\subsection{Vertical Pressure Difference along Immersion Nozzle}

The flow field near the immersion nozzle ${ }^{14)}$ and some of the symbols used in this model study are shown in Fig. 13, where $\sigma_{12}$ is the interfacial tension and $v_{1}$ and $v_{2}$ are the kinematic viscosities of the upper and lower liquid layers, respectively. The maximum pressure difference at the forward stagnation point of the immersion nozzle placed in the upper mold powder layer is denoted by $\Delta p_{\max 1}$ and the minimum pressure at around $\theta=\pi / 2$ and $\theta=3 \pi / 2$ of the nozzle in the molten steel layer is represented by $\Delta p_{\min 2}$.

$$
\begin{aligned}
& \Delta p_{\max 1}=C_{\text {pmax } 1} \rho_{1} V_{1}^{2} / 2 \\
& \Delta p_{\min 2}=C_{\text {pmin } 2} \rho_{2} V_{2}^{2} / 2
\end{aligned}
$$

where $C_{\mathrm{pmax} 1}$ and $C_{\mathrm{pmin} 2}$ are the maximum and the minimum pressure coefficients, $\rho_{1}$ and $\rho_{2}$ are the densities of molten mold powder and molten steel, $V_{1}$ and $V_{2}$ are the velocities of molten mold powder and molten steel flows approaching the cylinder, respectively. The subscripts 1 and 2 denote the upper and lower layers, respectively.

The pressure difference in the vertical direction, $\Delta p_{12}$, appearing behind the immersion nozzle, i.e., on the left hand side of the immersion nozzle in Fig. 13 is given by

$$
\begin{aligned}
\Delta p_{12} & =\left(\Delta p_{\max 1}-\Delta p_{\min 2}\right) \\
& =C_{\text {pmax } 1} \rho_{1} V_{1}^{2} / 2-C_{\text {pmin } 2} \rho_{2} V_{2}^{2} / 2
\end{aligned}
$$

According to the previous papers, ${ }^{4,14)}$ the following force balance holds for the liquid behind the immersion nozzle of good wettability:

$$
\Delta p_{12}+\rho_{1} g H_{\text {age }}=\rho_{2} g H_{\text {age }}
$$

where $g$ is the acceleration due to gravity, $H_{\text {age }}$ is the maximum penetration depth of the mold powder along the rear stagnation point of the immersion nozzle in the molten steel layer and the suffix $g$ means good wettability. The wettability is evaluated in terms of the contact angle.

Substitution of Eq. (7) into Eq. (8) yields:

$$
H_{\text {age }}=\left[C_{\text {pmax } 1} \rho_{1} V_{1}^{2} / 2-C_{\text {pmin } 2} \rho_{2} V_{2}^{2} / 2\right] /\left[g\left(\rho_{2}-\rho_{1}\right)\right]
$$

If the mold powder is stationary $\left(V_{1}=0\right)$, Eq. (9) reduces to

$$
H_{\text {age }}=-C_{\text {pmin } 2} \rho_{2} V_{2}^{2} /\left[2 g\left(\rho_{2}-\rho_{1}\right)\right]
$$

The maximum penetration depth, $H_{\text {age }}$, for the transient velocity profile shown in Fig. 3 therefore can be predicted 
by reading $C_{\mathrm{pmax}}$ and $C_{\mathrm{pmin}}$ values from Figs. 11 and 12 . Even if the velocity of a molten steel meniscus flow approaching the immersion nozzle in the real continuous casting mold is different from the profile expressed by Eq. (4), the maximum and minimum pressure coefficients can readi-

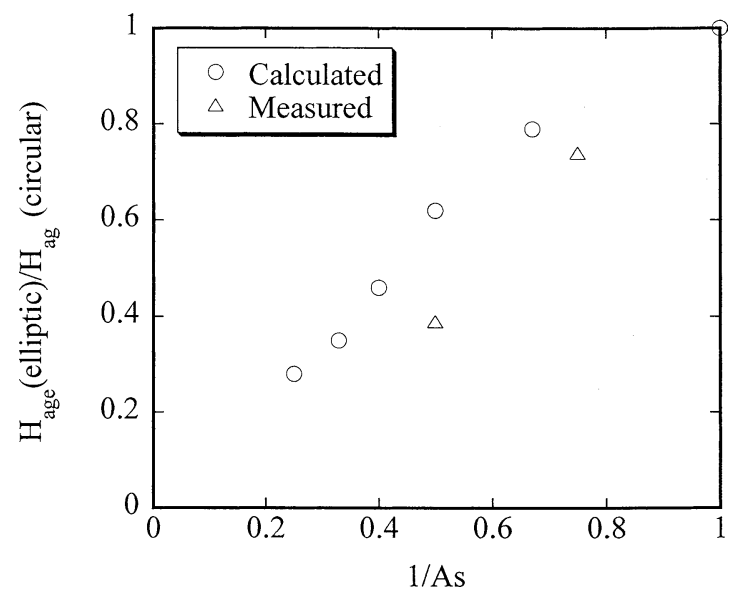

Fig. 14. Comparison of calculated and measured values of maximum penetration depth of silicone oil for approaching flow expressed by Eq. (4). ly be calculated by the vortex method.

Molten mold powder seems stationary in the real continuos casting mold. Accordingly, the mold powder entrapment is governed mainly by $C_{\text {pmin }}$, as suggested from Eq. (10). Figures 8 and 9 suggest that the effect of the velocity history of unsteady reversing flow appears significantly in the initial stage of reversing flow arrival.

\subsection{Comparison of Calculated with Measured Values of Maximum Penetration Depth}

Measurement of the maximum penetration depth of molten mold powder along the immersion nozzle is very difficult at present in the real continuous casting mold. As a

Table 1. Physical properties of liquids (298 K)

\begin{tabular}{|l|c|c|c|}
\hline \multicolumn{1}{|c|}{ Liquid } & $\begin{array}{c}\text { Kinematic } \\
\text { viscosity } \\
v_{1}, v_{2} \\
\left(\mathrm{~mm}^{2} / \mathrm{s}, \mathrm{cSt}\right)\end{array}$ & $\begin{array}{c}\rho_{1}, \rho_{2} \\
\left(\mathrm{~kg} / \mathrm{m}^{3}\right)\end{array}$ & $\begin{array}{c}\sigma_{12} \\
(\mathrm{mN} / \mathrm{m})\end{array}$ \\
\hline Salt water & 1.0 & 1013 & \\
\hline Silicone oil 2 & 2.0 & 873 & 52.7 \\
\hline Silicone oil 50 & 50 & 960 & 52.7 \\
\hline Silicone oil 100 & 100 & 965 & 53.0 \\
\hline
\end{tabular}

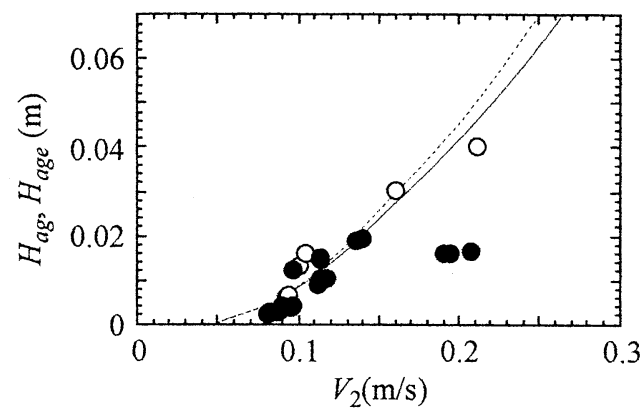

(a) Silicone oil 2

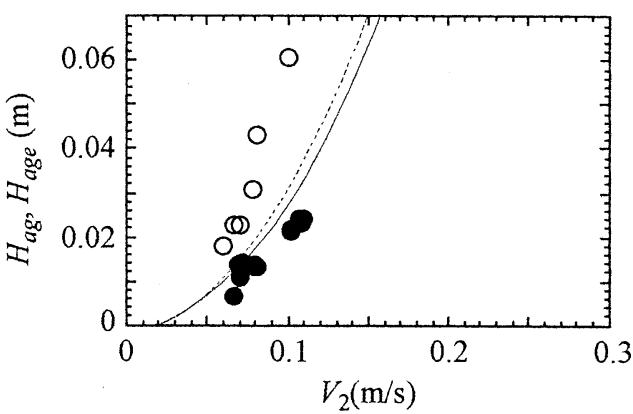

(b) Silicone oil 50

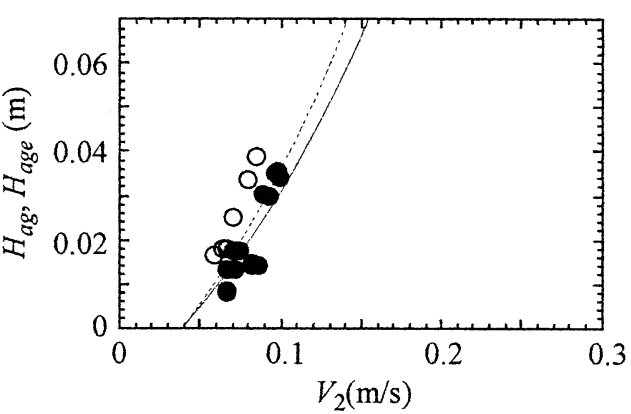

(c) Silicone oil 100

Fig. 15. Comparison between $H_{\mathrm{age}}$ and $H_{\mathrm{ag}}\left(D_{y} / B=D / B=0.3, D_{y} / D_{x}=0.75, A_{\mathrm{s}}=4 / 3\right.$, solid line: elliptic cylinder, broken line: circular cylinder) 
first step, numerical results were compared with the results obtained from cold model experiments with the velocity profile expressed by Eq. (4).

Figure 14 shows a comparison of the calculated values of $H_{\text {age }}$ with the measured values for the gradual acceleration of $V_{1}=V_{2}=0.08 \mathrm{~m} / \mathrm{s}$, where $H_{\mathrm{ag}}$ is the maximum penetration depth for a circular cylinder. Measurements were carried out using water and silicone oil as the working fluids. The measured values in Fig. 14 were obtained for a silicone oil of a kinematic viscosity of $100 \mathrm{~mm}^{2} / \mathrm{s}$. The physical properties of water and silicone oil are listed in Table 1. The details of the measurement method can be seen in the previous paper. ${ }^{4}$ Equation (9) slightly overestimates the measured values of $H_{\text {age }}$. Such a degree of discrepancy is acceptable in this kind of measurement. This figure means that the mold powder entrapment is highly suppressed with an increase in the aspect ratio of the immersion nozzle.

Figure 15 shows the calculated and measured values of $H_{\text {age }}$ and $H_{\text {ag }}$ against the approaching velocity of water, $V_{2}$. The aspect ratio, $A_{\mathrm{s}}$, was $4 / 3$. The velocity of silicone oil, $V_{1}$, is equal to $V_{2}$. The solid and broken lines denote the calculated values of $H_{\text {age }}$ and $H_{\mathrm{ag}}$, respectively. The calculated values can satisfactorily approximate the measured values for every silicone oil. Equations (9) and (10) state that the mold powder entrapment is influenced by the densities of molten steel and molten mold powder. Other physical properties such as the kinematic viscosity and interfacial tension may affect the mold powder entrapment. Further investigations using molten metals are required for clarifying the role of the physical properties.

\section{Conclusions}

The vortex method was used for calculating the maximum and minimum pressure coefficients, $C_{\mathrm{pmax}}$ and $C_{\mathrm{pmin}}$, around an elliptic cylinder. Information on the coefficients is required for designing an immersion nozzle of an elliptic shape, because they are closely associated with the penetration depth of molten mold powder along the immersion nozzle. An elliptic immersion nozzle of a larger aspect ratio was found to be more effective for preventing the mold powder entrapment.

\section{Acknowledgement}

The authors express their thanks to Dr. J. Yoshida and Mr. D. Iguchi for preparing some of the figures.

\section{Nomenclature}

$A_{\mathrm{s}}:$ Aspect ratio $\left(=D_{x} / D_{y}\right)(-)$

$C_{\mathrm{p}}:$ Pressure coefficient (-)

$C_{\mathrm{pmax}}:$ Maximum pressure coefficient $(-)$

$C_{\text {pmin }}:$ Minimum pressure coefficient (-)

$D_{x}, D_{y}$ : Long and short diameters of an elliptic cylinder (m)

$H_{\text {age }}$ : Penetration depth of silicone oil along elliptic cylinder of good wettability $(\mathrm{m})$

$H_{\text {ag }}$ : Penetration depth of silicone oil along circular cylinder of good wettability $(\mathrm{m})$

$p$ : Pressure $(\mathrm{Pa})$

$p_{\mathrm{s}}: \quad$ Static pressure $(\mathrm{Pa})$

$V:$ Velocity of flow approaching a cylinder $(\mathrm{m} / \mathrm{s})$

$V_{0}:$ Velocity of flow on the final stage $(\mathrm{m} / \mathrm{s})$

$\Delta p:$ Pressure difference $\left(=p-p_{\mathrm{s}}\right)(\mathrm{Pa})$

$\Delta p_{12}$ : Vertical pressure difference along an immersion nozzle $\left(=\Delta p_{\max 1}-\Delta p_{\min 2}\right)(\mathrm{Pa})$

$\rho:$ Density $\left(\mathrm{kg} / \mathrm{m}^{3}\right)$

Subscripts

1: Upper liquid layer

2: Lower liquid layer

\section{REFERENCES}

1) S. Taniguchi and A. Kikuchi: Tetsu-to-Hagané, 78 (1992), 527.

2) Jpn. Soc. Prom. Sci., 19th Committee: Recent Development in Studies of Non-metallic Inclusions in Steel, Jpn. Soc. Prom. Sci., Tokyo, (1994).

3) M. Iguchi: Fluid Flow in Continuous Casting Mold and Behavior of Non-metallic Inclusions, ISIJ, Tokyo, (2003).

4) J. Yoshida, M. Iguchi and S. Yokoya: Tetsu-to-Hagané, 87 (2001), No. 8, 529

5) J. Yoshida, M. Iguchi and S. Yokoya: Tetsu-to-Hagané, 88 (2002), No. 5, 264

6) M-H. Chou and W. Huang: Int. J. Numerical Methods in Fluids, 23 (1996), 711.

7) S. F. Hoerner: Fluid-Dynamic Drag, Published by the author, (1965).

8) R. D. Blebins: Applied Fluid Dynamics, Van Nostrand Reinhold Co., New York, (1984).

9) K. Kamemoto: J. Jpn. Soc. Comp. Fluid Dynamics, 2 (1993), No. 1, 20.

10) K. Kamemoto: J. Jpn. Soc. Comp. Fluid Dynamics, 2 (1994), No. 2, 28.

11) T. Kida, H. Ueda and M. Kimura: Computational Fluid Dynamics J, 9 (2000), No. 2, 64.

12) Y. Ueda and T. Kida: Computational Fluid Dynamics J., 11 (2002), No. 2, 213.

13) H. Schlichting: Boundary-Layer Theory, 7th ed., McGraw-Hill, New York, (1979), 425.

14) J. Yoshida and M. Iguchi: Tetsu-to-Hagané, 87 (2001), No. 12, 741. 